\title{
3D A Retrospective Cohort Study Examining 3D Reconstruction to 2D Radiology in Identifying Rib Fractures
}

\author{
Gordon McCauley ${ }^{1}{ }^{*}$, Sanjeet Singh ${ }^{2}$, Sudeep Das De ${ }^{2}$, Alan Kirk ${ }^{2}$ \\ ${ }^{1}$ College of Medical, Veterinary and Life Sciences, University of Glasgow, Glasgow, United Kingdom \\ ${ }^{2}$ Department of Cardiothoracic Surgery, Golden Jubilee National Hospital, Glasgow, United Kingdom
}

Email address:

2285622M@student.gla.ac.uk (G. McCauley)

*Corresponding author

\section{To cite this article:}

Gordon McCauley, Sanjeet Singh, Sudeep Das De, Alan Kirk. 3D A Retrospective Cohort Study Examining 3D Reconstruction to 2D Radiology in Identifying Rib Fractures. International Journal of Cardiovascular and Thoracic Surgery. Vol. 3, No. 5, 2017, pp. 41-52. doi: $10.11648 /$ j.ijcts.20170305.12

Received: February 22, 2017; Accepted: March 22, 2017; Published: October 23, 2017

\begin{abstract}
Introduction: Rib fractures are common presentations at emergency departments across the UK. $21 \%$ of emergency admissions are related to rib fractures, resulting from up to $39 \%$ of all blunt-trauma to the chest. Imaging is essential for identification of rib fractures but also for planning potential surgical intervention. The introduction of 3D imaging has enhanced the pre-surgical planning in many fields of medicine. We compared 3D CT in the identification of rib fractures to conventional 2D radiology. Methods: A retrospective cohort study of 22 patients was conducted at a district general hospital between Novembers 2014-2016. Chest X-ray, 2D CT and 3D CT findings were compared for the number of rib fractures and identification of displacement of rib fractures. Statistical analysis was performed using a 3-way ANOVA, with Cohen's kappa statistic $(\kappa)$ to identify agreement. There were 22 patients (20M: $2 \mathrm{~F}$ ) patients in this study. The mean age of patients was 50.2 \pm 21.7 (range 10-94). Chest X-rays had the poorest identification rate with slight agreement with $3 \mathrm{D} C \mathrm{CT}(\kappa=0.018)$. There was also a slight agreement for identification of displacement between 2D and 3D CT imaging $(\kappa=0.127)$ Conclusion: Chest X-ray on its own is inferior to $2 \mathrm{D}$ and $3 \mathrm{D}$ CT. 3D CT adds to the value of 2D imaging as it gives an enhanced view of any possible fracture displacement.
\end{abstract}

Keywords: Rib Fractures, 3D Imaging, Chest X-ray

\section{Introduction}

Rib fractures are a common injury seen throughout every emergency department across the UK, from major trauma centres to small district general hospitals. It is a condition that can cause much morbidity and even death to its sufferers. Rib fractures are classified into a wide spectrum, ranging from simple single fractures of one rib to the more serious flail chest of multiple, comminuted fractures [1]. $21 \%$ of emergency admissions are related to rib fractures [2] with $39 \%$ of all blunt-trauma to the chest leading to fracturing of a rib [3]. It is therefore evident how rib fractures are the most common chest injury today [3].

There are considerable complications associated with rib fractures that can affect people of any age. These complications can range from pneumonia to more lifethreatening conditions such as acute respiratory disease [4], liver laceration and even death. The mortality rate for flail chest is as high as $25 \%$ [4], and fractures of more than six ribs leads to increase of mortality rate to $34 \%$ [3]. There is significant morbidity associated with rib fractures ranging from enduring pain, breathing difficulties and deformity, not only in the acute stage of the injury but also chronically. It has been reported [1], that a rib fracture can significantly affect the quality of life of the sufferer even 24 months after the insulting injury. This is highlighted by the low rate of return to work from sufferers, which shows the clear effect this injury has both for the patient and in its cost to society [1].

The morbidity and mortality caused by rib fractures are 
due to the pathology that a disruption to the rib cage causes [5]. The fracturing of a rib interferes with the normal ventilatory function of the chest wall. The trauma to the chest leads to an inflammatory, oedematous reaction within the chest wall and lungs themselves, which causes interference in gaseous exchange. Similarly, due to the disturbance in the mechanics of the rib cage there is failure in the ventilation and a reduction in efficient breathing. This leads to the complications of atelectasis and retention of secretions that leads to the risk of pneumonia. This inefficiency in ventilation can be exacerbated by the presence of a flail segment. This causes dysfunction because as the rib cage travels outwards during inspiration, the flail segment paradoxically travels inwards further reducing ventilation. These pathologies lead to the various complications associated with rib fractures and this highlights the necessity for prompt treatment of this condition to avoid these risks.

There have been substantial advancements in the treatment of rib fractures over the previous century [6]. Initial research into the treatment of rib fractures divided it into two main groups: internal support techniques and external support techniques. Internal supportive techniques involved the use of mechanical ventilation for the supportive therapy of these patients, it was first reported in 1901 [7] however it took nearly 50 years for it to become part of the modern regime of rib fractures that is still used today. The ventilation varied from intermittent or continuous mechanical ventilation to ventilation with tracheostomy to assisted-breathing with the addition of a mouthpiece. Sll therapy aimed at the same basic principle: to increase lung function by supporting the respiratory and pulmonary muscles and increasing lung capacity.

External support like internal support dramatically altered over the $20^{\text {th }}$ century. It was first reported in 1928 by Jones [8] with the use of percutaneous bullet forceps followed by traction to stabilize the fractured ribs. There were various publications from 1941-45 that highlighted the use of nonoperative external support in the therapy of rib fractures. These techniques included the use of tape strapping and placement of sandbags on patients to provide support and to try and reduce the risk that invasive procedures potentiated. Over this time, the invasive external techniques for rib fracture stabilization have advanced from Jones' first publication. The instruments and materials utilised progressed from using clothes hangers as reduction forceps to titanium clips or titanium plates used commonly today [9].

These techniques coupled with mechanical ventilation became the mainstay for external rib fracture treatment until the invention of invasive suspension of the fractured ribs with or without open reduction towards the end of the $20^{\text {th }}$ century. Again, the materials used have changed over the previous decades from simple sutures and K-wires, to the more sophisticated intramedullary devices and metal titanium plates that are more commonly used. 2008 provided the launch of dedicated "systems" for the stabilization of rib fractures, which consist of all the materials and instruments needed for rib fixation. These "systems" help to provide a standardization of materials and techniques used across the UK.

However even in present day there is still no definitive algorithm for rib fractures and flail chest treatment. The management pathways follow two main groups, those that require supportive therapy and those who require interventional treatment. The focus for supportive therapy involves adequate analgesia and physiotherapy. The analgesia follows a similar pain management scale to most analgesic pathways. Initially simple analgesics such as paracetamol and non-steroidal anti-inflammatories may be used, however generally stronger painkillers are required for the pain with opioids mainly used. Interventional methods may also be required such as regional anaesthetic techniques to paravertebral blocks and epidural anaesthesia [5]. Physiotherapy has also played a dramatic role recently in improving patient's pulmonary outcomes and reducing morbidity [5]. The role of these supportive therapies is to alleviate the painful breathing for these patients, which will lead to a reduction in the respiratory complications. Interventional management consists of ventilatory management and surgical fixation. Both methods as previously described have changed radically over the previously century. Currently ventilatory management includes continuous positive airway pressure, but in more severe cases may require invasive ventilation in an Intensive Care Unit (ICU). Surgical fixation is normally used in more severe cases of rib fractures; this includes cases of flail chest that are intubated or physiologically compromised or cases where there is respiratory failure or where previous therapies have failed [5].

This uncertainty in following a clear treatment pathway for rib fractures may be based on the poor availability and pool of randomized controlled trials around this subject matter. A recent meta-analysis published in August 2016 [10] attempted to investigate the outcomes of operative techniques in the treatment of rib fractures in comparison to nonoperative techniques. The literature review analyzed three randomized control trials investigating operative versus nonoperative therapy. Their primary outcomes were to quantify the mortality and pneumonia rate in these patients and secondary aims where to look at the mechanical ventilation time, time in ICU and hospital and then to assess need for tracheostomy. What was found was that operative techniques showed no difference in mortality rates but significantly reduced the rate of pneumonia, time on ventilator, tracheostomy rate and stay in ICU and hospital overall. Though there are limitations due to only a small number of studies being analyzed, the meta-analysis showed the benefits and positive outcomes provided by operative management of rib fractures. This then highlights the importance of adequate radiology and preparation for these surgical procedures.

The role of radiology plays a crucial role in not only the identification of rib fractures but also in the surgical preparation for any possible therapy. Like the treatment of rib fractures, there is still an uncertain radiological pathway for the diagnosis and assessment of rib fractures. Livingston et al 
(11), in 2007 analysed the role of CT imaging compared to plain radiograph in reducing the mortality rate and rate of acute respiratory distress. Though they failed in proving their hypothesis they did highlight some important points. They found that entirely relying on plain radiographs alone for the diagnosis would miss rib fractures in over $50 \%$ of cases and they identified that $\mathrm{CT}$ imaging on average found three extra rib fractures compared to plain chest radiographs. However, they found also that up to $43 \%$ of CT reports inaccurately reported the number and location of the rib fractures, and in many of these reports the term "multiple rib fractures" were aimlessly used. They concluded that plain radiograph held an important screening role in the identification of rib fractures but that CT imaging provided a higher sensitivity and provided a clearer visualisation of the chest. This paper shows the radiological difficulty in the identification of rib fractures and their position and the need for further research into new techniques of visualising rib fractures.

Future research must consider the complicated 3D anatomy of the chest wall and the limitations that $2 \mathrm{D}$ imaging has in identifying a disruption to the rib cage. There has been much recent research into the role of $3 \mathrm{D}$ imaging in visualisation of fractures in bones throughout the body [11], but there is a lack of evidence into the role of 3D imaging of rib fractures. With the $3 \mathrm{D}$ component of its anatomy, this form of radiology should provide additional benefit. Pulley et al [3] is one of the few studies analyzing the role of 3D imaging compared to $2 \mathrm{D}$ radiology. The aim of the study was to assess the reliability of both radiological techniques and to assess if there was a change in surgical plan or route with the addition of 3D imaging. They found that both $2 \mathrm{D}$ and 3D CT imaging provided excellent reliability in identifying rib fractures but that 2D imaging showed a higher reliability in identification compared to 3D. Of importance they found that the addition of 3D imaging would have changed the surgical route in $65.7 \%$ of cases previously based on $2 \mathrm{D}$ imaging alone. This study shows the advantages of the use of $3 \mathrm{D}$ imaging in rib fracture radiology, however the paper only analyzed the number of rib fractures present but not the location nor displacement of these fractures which one could hypothesize would be more clearly delineated in 3D imaging and which holds greater weight in the treatment of the patient.

3D imaging has advanced substantially in recent years and the development of 3D model production has only added to the potential benefits of this visual technique. The role of $3 \mathrm{D}$ modeling has revolutionized many specialties in medicine and added greater diagnostic accuracy and enhanced surgical pre-operative planning. The use of $3 \mathrm{D}$ imaging and prototyping has become a mainstay in the maxillofacial surgery preparation [11] [13] where 3D modeling has shown to improve diagnostic and surgical approach to many of these operations. Similarly, in orthopaedics, this technology has been used to visualise the anatomy of the spine, hip and shoulder and in many cases has helped surgeons plan the exact areas of screw insertion preoperatively. Due to the similarities of these surgeries to rib fixation, this technology and visual technique should only enhance surgical approach and more importantly improve clinical outcomes for those patients involved.

Aims

Primary Aim

To assess the accuracy of 3D CT imaging in identifying and quantifying rib fractures compared to 2D radiology.

Secondary Aim

To assess the accuracy of 3D CT imaging to identify displaced rib fractures in comparison to $2 \mathrm{D}$ radiology.

\section{Materials and Methodology}

This is a retrospective cohort study analyzing the identification and assessment of rib fractures from radiology of patients who suffered from rib fractures between the times of November 2014 until November 2016 in the Forth Valley Royal Hospital.

Patient Selection

Inclusion Criteria

a) Patient of any age.

b) Patient who had a confirmed diagnosis of rib fracture.

c) Patient who had both a valid chest X-ray, 2D CT scan available related to the potential rib fracture diagnosis.

Exclusion criteria

- Patients that the diagnosis of rib fracture was not confirmed with radiology.

- Patients that did not have a chest X-ray and/or 2D CT scan available to be viewed.

Information that was collected included:

a) Patient Age.

b) Patient Gender.

c) Mechanism of injury leading to rib fracture.

d) Date of injury occurrence.

e) Date X-ray was taken.

f) Date CT was taken.

g) Number of rib fractures, position and displacement of fracture reported by X-ray.

h) Number of rib fractures, position and displacement of fracture reported by $2 \mathrm{D}$ CT.

i) Number of rib fractures, position and displacement of fracture reported by 3D CT reconstruction.

j) Any further complications and mortality reported due to the injury.

\subsection{Data Collection}

In November 2016 data was collected from the Picture Archiving and Communication System (PACS), (Version 11.4.1.1102, Carestream Health Inc (independent subsidiary of Onex Corporation, Toronto, Ontario, Canada)) in the Golden Jubilee Hospital using patients' CHI (Community Health Number, an unique patient identifier) number provided by Forth Valley Royal Hospital. PACS is used throughout Scotland to allow information on patients to be passed effectively and efficiently to and from hospitals and doctors throughout Scotland. This list had been made by searching the Forth Valley Royal Hospital database for 
patients with "rib" and "blunt trauma" in their diagnosis that had been admitted to the A\&E department between the dates November 2014 and November 2016. This patient list contained $63 \mathrm{CHI}$ patients' numbers. Each individual $\mathrm{CHI}$ patient number provided was searched on the PACS system in the Golden Jubilee Hospital where the project was based. From PACS the chest X-rays and axial 2D CTs (Discovery CT750 HD, GE Healthcare 64 slide scanner dual source) of the patients in this list could be viewed. 2D CT was then converted into a $3 \mathrm{D}$ reconstruction view of the $2 \mathrm{D}$ axial image. The number, the position and the displacement of the rib fractures that were reported in the X-rays and 2D CTs from the radiologist's report of each individual patient were retrospectively recorded. After converting the CT into the 3D reconstruction view, it was then assessed by the same cardiothoracic surgeon for each patient, where the number, location and potential displacement of any rib fractures where recorded. The cardiothoracic surgeon was blinded to the radiological results of the previous chest X-ray and 2D axial CT scans and to the patients' identity. A cardiothoracic surgeon was chosen to analyse the $3 \mathrm{D}$ reconstruction of the CT scans, as this will be the group of medical professionals that may be potentially utilising the $3 \mathrm{D}$ reconstruction of the $2 \mathrm{D}$ scans. The numbers of fractures were recorded numerically, the position of the fractures was recorded as either anterior, lateral or posterior and the displacement of the rib fractures were recorded as either "Yes" or "No". Demographic information included the information recorded as mentioned above. This information was collected from PACS and any further complications from the injury were recorded from the 2D CT scans' radiological reports for each patient. The demographics of the area were recorded using the Scottish Public Health Observer Profiles Tool provided by NHS Scotland. Information was recorded onto an Excel spreadsheet.

\subsection{Statistical Analysis}

The average rib fractures were analysed for each imaging modality, a range, a standard deviation and $95 \%$ confidence interval were also reported for each imaging technique. The number of fractures recorded for each modality was analysed with a Student's t-test and/or an analysis of variance where appropriate. Agreement was assessed between the different methods of imaging in detecting number and potential displacement of fractured ribs using Cohen's kappa statistics. Bland-Altman Plots were used to visualise the agreement and potential bias between the imaging techniques in detecting fractures. These statistics were produced using SPPS (SPPS Version 22 for Windows).

\section{Ethics and Confidentiality}

The list of patients' numbers who were applicable for the project was printed out and numbers were assigned to each patient for identification. The data was documented in an anonymised manner into an Excel spreadsheet with the assigned number used as an identifier, with no patient information leaving the Golden Jubilee Hospital. This project was ethically carried out as it did not intervene with any of the patients' wellbeing or autonomy and would ultimately benefit the patients included.

\section{Results}

\section{Demographics}

There were 63 patient numbers selected from the search. Of these 63 patients only 22 were eligible for the study due to either not having confirmation of a rib fracture from radiology or unavailability of X-ray and/or CT scans to view. Of this sample, there were 20 males and 2 female patients. The average age of the patients was 50.2 with an age range of 10-94 and with a standard deviation of 21.65. The mechanism of injury was also recorded with 13 out of the 22 patients suffering rib fractures due to road traffic collision $(59.1 \%), 8$ patients suffering fractures due to a fall $(36.4 \%)$ and 1 patient suffering from rib fractures due to assault (4.5\%). The demographic area of Falkirk where the Forth Valley Royal Hospital is situated was analyzed in comparison to Scottish averages. This area maintained a similar male and female life expectancy to the Scottish average (76.9 for males and 80.6 for females). The socioeconomic landscape differed to the Scottish average with a population-income deprived percentage of $12.7 \%$ in comparison to Scottish average of $13.1 \%$. Interestingly there was a lower rate of road traffic accident casualties seen in the Falkirk area compared to the national average, with an average of 47.6 per 100,000 of population in comparison to the national average of 58.9 per 100,000 of population.

\section{Number of Fractures}

It was seen that the average number of rib fractures identified for chest X-rays was 1.81 (95\% CI- 0.282-3.354 and a range of 0-6), for 2D CT imaging was 6.13 (95\% CI4.601-7.672, with a range of 1-15) and for 3D CT imaging was 5.32 (95\% CI- 3.782-6.854, with a range of 0-15).

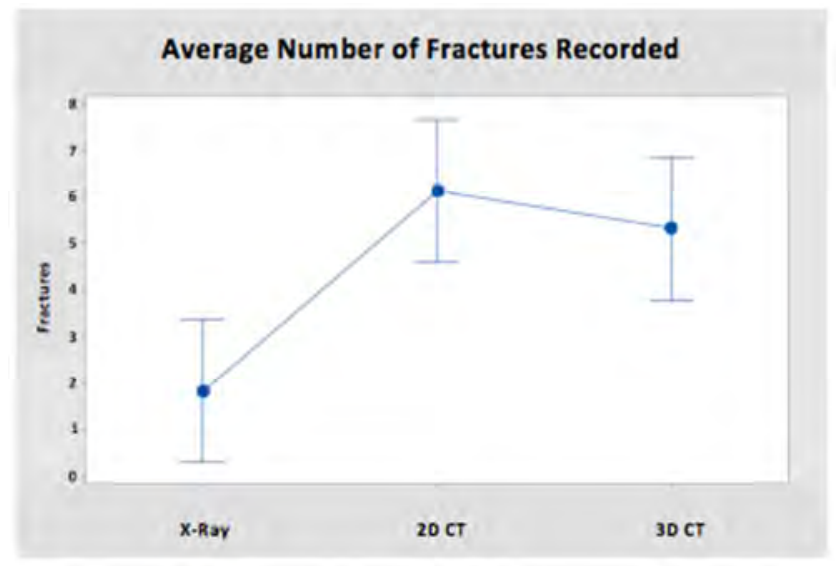

Figure 1. Average Number of Fractures Recorded. Blue dots represent the average number of rib fractures for each imaging modality with the intervals representing the $95 \%$ confidence interval of each average. Chest X-rays average number detected was 1.81 (95\% CI, 0.282-3.354), 2D CT imaging 6.13 (95\% CI, 4.601-7.672) and for 3D CT imaging 5.32 (95\% CI, 3.7826.854). A p-value of $<0.0001$ produced using analysis of variance (ANOVA).

$40.9 \%$ of chest X-rays that did not detect any rib fractures 
were found to have rib fractures on $\mathrm{CT}$ scans and were classified as normal in the initial chest X-ray radiological report. Only in one patient $(4.55 \%)$ did all three modalities detect the same number of rib fractures (6 fractures each).

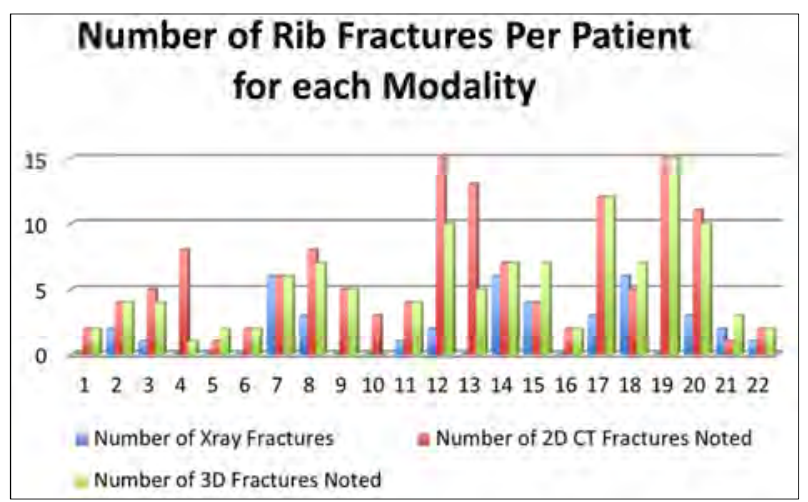

Figure 2. Displays the number of fractures detected for each individual patient by chest X-Ray, 2D CT and 3D CT respectively. Chest X-ray is labeled colour Blue, 2D CT labeled colour Red, 3D CT labeled colour Green. The $X$-axis displays each patient number and the $Y$-axis represents the number of rib fractures for these patients.

A Student's T-test was carried out between the imaging modalities to assess if there were significant differences between the results gathered (Appendix 2). Paired T-test demonstrated a T-value of -4.60 and a p-value of $<0.0001$ between chest X-ray and 3D CT therefore we can conclude that the null hypothesis can be excluded. However a paired T-test between 3D and 2D CTs produced a T-value of 1.42 and a p-value of 0.170 , therefore we cannot exclude the null hypothesis.

\section{Location of Fractures}

The locations of rib fractures were also recorded and calculated with 10 anterior rib fractures, 8 lateral rib fractures and 4 posterior rib fractures recorded.

\section{Location of Rib Fractures}

Anterior \& Lateral Posterior

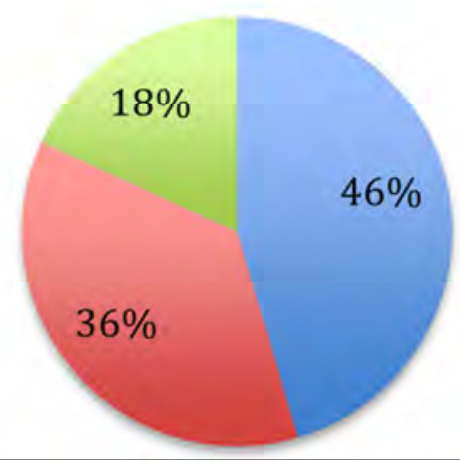

Figure 3. Shows the percentage of location of rib fractures for each patient (22 patients) recorded in the study. With 10 patients suffering from anterior fractures (46\%), 8 from lateral fractures (36\%) and 4 from posterior fractures $(18 \%)$.

\section{Displacement of Fractures}

3D CTs identified displacement in 12 out of the population of 22 patients, while 2D CT identified 6 out of 22 displaced fractures. 3D CTs identified all the displacements that were detected in 2D CTs. Chest X-rays did not detect any displaced rib fractures.

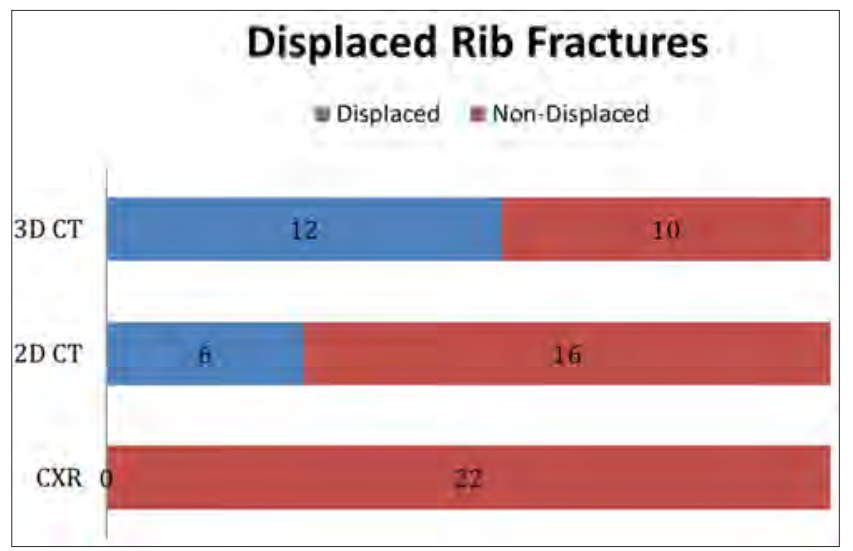

Figure 4. Displays the number of displaced rib fractures detected per modality. 3D CT detected displaced rib fractures in 12/22 of patients; $2 D C T$ detected 6/22 patients; chest X-Ray 0/22 patients. This shows 3DCTs increased ability to detect displacement of rib fractures. $C X R=$ Chest $X$-ray.

\section{Further Complications}

Complications that were acquired from the injury that caused the rib fractures were also recorded. It was seen that 10 out of the 22 patients suffered from a pneumothorax (45.5\%) with 2 out of the 22 patients $(9.1 \%)$ suffering from either haemothoraces, surgical emphysema, splenic laceration or pulmonary contusion. There was also a mortality rate of 2 out of the $22(9.1 \%)$ patients related to the injuries and complications sustained.

Assessment of Agreement

Kappa statistics were carried out to assess the agreement between the identification and assessment of rib fractures from 3D CT imaging and X-ray and between 3D CT imaging and 2D CT imaging. Bland-Altman plots were also produced to visualise the agreement between these modalities.

3D CT versus X-ray

The kappa coefficient produced was 0.018 . This shows that there is very poor agreement between $3 \mathrm{D} C \mathrm{C}$ versus $\mathrm{X}$ ray in the use of identification of rib fractures. This coefficient shows that there is a large risk of chance between both of these modalities gaining the same identification of rib fractures for a single patient. This is a poor agreement and opens the possibility of bias between the two imaging techniques.

3D CT versus 2D CT

The kappa coefficient produced was 0.447 . This shows that there is moderate agreement between the modalities and means that there is less of a risk of chance of both modalities identifying the same amount of rib fractures for a single patient compared to $3 \mathrm{D} \mathrm{CT}$ and X-rays. Ideally a reflection of substantial agreement would have a kappa coefficient greater than 0.6 [14] 


\section{Bland-Altman Plot: 3D CT versus X-ray}

The graph shows the relationship between the differences in modalities to the mean differences of both imaging techniques. The graph shows that there is a bias of 3.75 between 3D imaging and X-ray, or in other words that 3D CT imaging will identify or overestimate 3.75 more ribs than $\mathrm{X}$ rays. It is also important to note that as the mean rib fractures noted increases along the $\mathrm{x}$-axis that the difference between the fractures recorded substantially increases. This shows that 3D imaging appears to show a greater ability to identify rib fractures than X-ray, especially with an increase in the number of rib fracture.

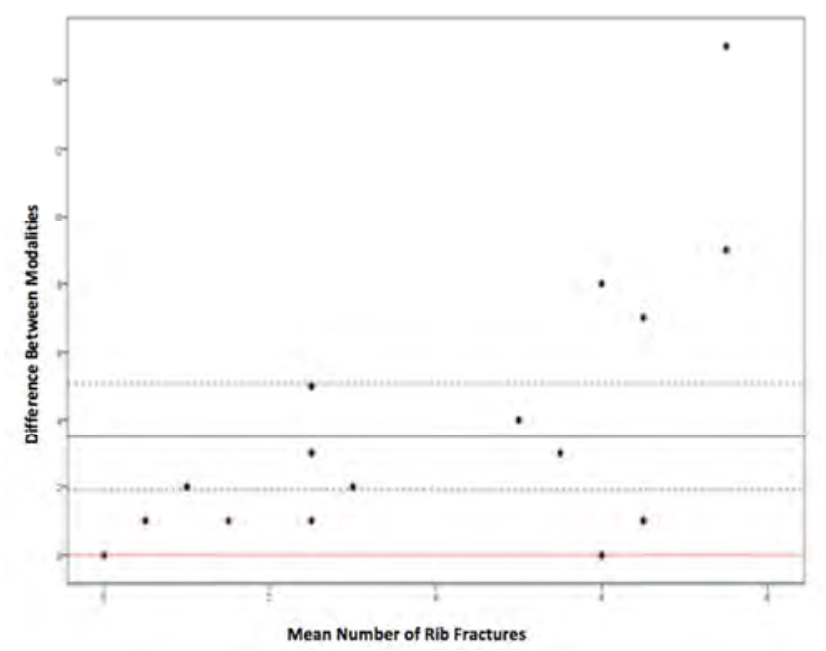

Figure 5. Bland-Altman Plot: $3 D$ CT versus $X$-ray. This image displays the agreement between $3 D C T$ and Chest X-ray. The average of the differences between the modalities is 3.75 (bold, black line on graph), with a standard deviation of 0.8 (dashed line), which deviates from the 0 mark (the red line). As the average number of rib fractures increases there is a greater difference in the abilities of the modalities to identify the number of rib fractures.

\section{Bland-Altman Plot: 2D CT versus 3D CT}

This graph shows the agreement between the two measurements of rib fracture identification. The average of the differences between the two modalities was 0.8 . There is no prominent change in this relationship with the increase of average rib fractures in comparison to imaging by X-rays. There are some outliers seen in the plot that cannot be accounted for and may explain the slightly lower than expected kappa coefficient, however the agreement seen between two imaging modalities is largely similar and shows approximately the same ability to identify rib fractures for a single patient.

Detection of Displacement between 3D and 2D CT

Kappa statistics were created to measure the agreement between $3 \mathrm{D}$ and 2D CT for identifying displacement. A kappa coefficient of 0.127 was produced which shows only slight agreement between the modalities. This shows that there is potential bias between the two imaging techniques and it is clear that 3D CT detects a higher amount of displacement compared to 2D CT.

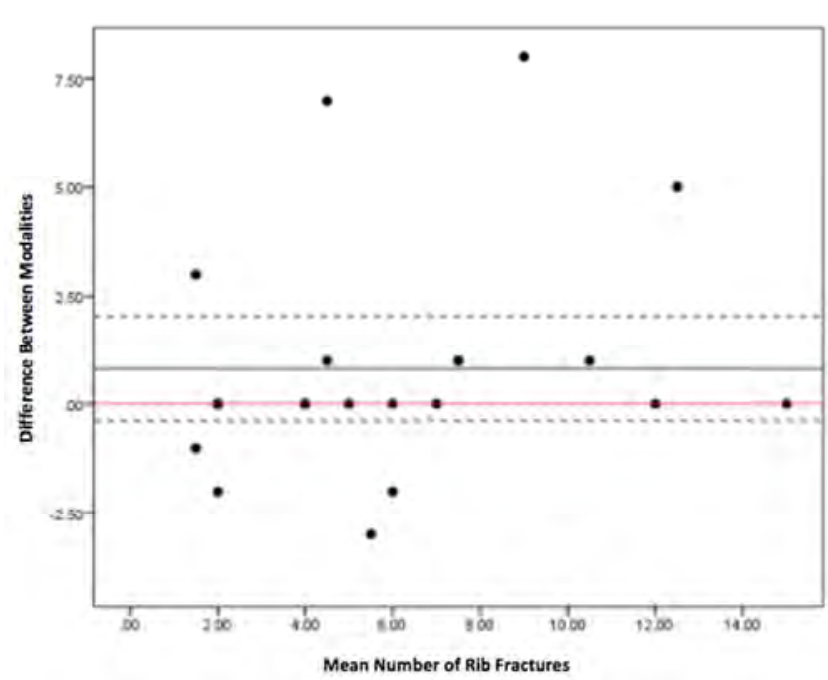

Figure 6. Bland-Altman Plot: $2 D C T$ versus $3 D C T$. This graph shows the agreement between the $2 D C T$ and $3 D C T$. The average of the differences between the two modalities was 0.8 (bold, black line on graph) with a standard deviation of 1.2 (dashed line). The average difference is closer to the 0 difference line (red line), this shows that there is greater agreement in comparison to $3 D C T$ versus chest $X$-ray.

\section{Discussion}

Rib fractures are a commonly occurring injury that can severely affect the lives of the injured parties. It has been associated with much morbidity and mortality not only due to the acute clinical sequelae of the injury itself but also due to the time off work and chronicity associated with the pathology at hand [1]. It is common that many patients suffer from prolonged pain commonly lasting greater than 8 weeks [15]

The chest wall possesses a complex architecture made up of varying structures. Its foundations are built around the twelve ribs that unite the sternum anteriorly to the vertebrae posteriorly $\mathrm{C}$ The ribs are supported by numerous layers of overlying muscle as seen in the abdominal wall. The innermost muscles consist of the intercostal muscles, which are found externally and internally to the ribs with a further innermost muscle behind the internal intercostal. The intercostal neuro-vascular bundle, consisting of the intercostal vein, artery and nerve, run between the internal and innermost intercostal muscle in the costal groove of the rib, which provides protection to these structures. Overlying these structures are the pectoral girdle muscles. These include the pectoralis major and minor anteriorly and the latissimus dorsi, levator scapulae, rhomboids and trapezius posteriorly. The complexity of the chest wall is a challenge for the surgical operations being undertaken around these structures. The aim of minimal muscle disruption as already mentioned is a key goal for many thoracic surgeons as well as protection of the neuro-vascular bundle, therefore it is crucial that imaging provides an accurate depiction of the human anatomy but also allows preparation for the site of incision and approach to be made to avoid disruption to the various muscle layers of the rib. 
There has recently been a change in the approach to the treatment of rib fractures. As previously mentioned the traditional therapy of analgesia with or without mechanical ventilation with little surgical input has changed over the last decades. This change has steered the therapy for multiple displaced fractures more towards the surgical route using rib fixation. This therapy has been enhanced by the introduction of the rib fixation packs that are used in many hospitals throughout the UK. Though this technique has been present for numerous years it is only being used intermittently due to the lack of research around its success and clinical outcomes. A meta-analysis of 11 papers examining surgical fixation versus non-operative management of flail chest found that there were notable differences between the two therapies, stating that surgical fixation was associated with much lower days on ventilation, days in the ICU and hospital as well as lower complication rates [17]. However the papers in the meta-analysis similar to many others on this topic consisted of retrospective studies with small sample sizes, which may leave itself open to bias. This has been an issue for the standardization of this surgery into the treatment of rib fractures as many surgeons are uncomfortable with the lack of randomized control trials into this surgical procedure [18].

However, in recent years two randomized control trials have analyzed the benefits of surgical rib fixation compared to non-operative methods. Marasco et al [19] carried out a prospective randomized study assessing the role of rib fixation in the treatment of traumatic flail chest. This study consisted of forty-six patients randomly split to receive either non-operative care or rib fixation. It was found that there were improved clinical outcomes for the patients who underwent surgical rib fixation and that there was notable cost effectiveness associated with surgical therapy. Though this paper shows the advantages of using rib fixation it takes only into account patients with traumatic flail chest, with the paper defining flail chest as "3 or more consecutive ribs fractured in more than 1 place, producing a free-floating segment of chest wall" [19]. This treatment therefore is not fully applicable to all cases of rib fractures but only those with multiple comminuted fractures. A similar study was carried out in 2016 [17]. again assessing surgical rib fixation versus medical treatment. This study included more types of rib fractures than flail chest but mainly focused on severe cases of multiple rib fractures. They again found that markedly lower rates of complications and greater pulmonary function were seen in surgical rib stabilization in comparison to medical management.

As surgical stabilization is being gradually more accepted into the standard care of multiple rib fractures, there has been research into a new minimally invasive technique to minimize disruption to the normal anatomy. A case report in 2014 outlined their experience with using thoracoscopyassisted minimally invasive technique for rib fixation. The case report analysed three patients who were suffering from anterolateral flail chest and they found that this technique provided equal stabilization as before but that it was associated with far less operation time, with all patients experiencing a more rapid recovery [20]. It was in this case report however that for each patient 3D imaging was used pre-surgically. It was used as an imaging technique for the diagnosis of the rib fractures, however interestingly, it was used as a modality to bend the prostheses and bars being used into the correct position before being placed by a minimally invasive technique into thorax to stabilize the rib fracture. This potentially shows the advantage and benefit that $3 \mathrm{D}$ imaging can add to the standard radiology currently used. It possesses the ability to identify pathology within the thorax but also allows pre-surgical preparation, from the incision site to the angulation of the prostheses used to be carried out before initiating the operation. This is important as surgical fixation of rib fractures like other surgical specialties are taking on a more minimally invasive approach. It is important that there is an imaging modality that can complement the emergence of this new surgical thinking.

This evidence shows the inherent benefits of using the surgical approach in the treatment of severe multiple rib fractures however these papers do not take in account the complexity and skill required for this surgical approach. There is a lack in knowledge of these surgeries and the prostheses and equipment required for them. The surgical techniques and approaches are different for each position of the fracture being stabilized. This means that for each position of fracture; anterior, lateral, posterior or more proximal or distal, there are different incision sites and different routes for the surgical fixation [2]. This coupled with the fact that certain ribs require specific prostheses. It is therefore crucial for the operating surgeon to know and understand the disruption to the normal anatomy. This ultimately means that knowledge not only of which rib is being affected must be known, but also the exact displacement and angulation of the fractured ribs must be known prior to commencing the surgery.

From the results gathered from this project 3D CT possesses a superior ability to provide greater visualisation of the fractured rib. It is seen that $3 \mathrm{D}$ imaging is far superior in identifying rib fractures than chest X-ray with a higher mean rib fracture detected by $3 \mathrm{D}$ imaging to chest X-ray. There was also a very poor agreement between both modalities especially as the number of fractures increased a greater discrepancy between the two modalities was seen. This highlights that chest X-ray alone is poor detector of rib fractures compared to $3 \mathrm{D}$ CT particularly when multiple fractures are suspected. There is less of a discrepancy between the numbers of rib fractures detected by $3 \mathrm{D}$ versus 2D CT with a moderate agreement between the two modalities. This shows that both imaging techniques provide a similarly equal ability to detect any rib fractures even if larger numbers of fractures are suspected. It is the lack of agreement between the two modalities for detecting displacement of these fractures that is more meaningful. There is very poor agreement between $3 \mathrm{D}$ and $2 \mathrm{D}$ CT for detection of displacement, with 3D detecting a far greater number of displaced rib fractures than 2D CT. This is extremely important if surgical intervention in particular 
when rib fixation is required. As previously mentioned it is imperative to understand the position and angulation of the rib fractures, as these weighs heavily on which surgical approach and prostheses may be used, by the operating surgeon. This project strongly supports the recommendation that for chest wall fixation surgery after rib trauma that using $3 \mathrm{D}$ imaging provides greater information of the affected area for surgery and would be necessary for pre-surgical assessment.

There has been emerging research into the use of 3D printing for surgical applications, which may have uses for the detection and treatment of rib fractures. 3D CT imaging provides a cheap and effective way of visualising the anatomy of the body however a 3D model may provide further benefits in the planning for thoracic surgeons before surgical rib stabilization [20]. This project provided the opportunity to print a model of one of the patients included within the study. A patients CHI number was chosen at random and this was the model thorax that was printed. Information on the machine used is found in Appendix 1 . Once the model was printed, a cardiothoracic surgeon was then asked to detect the number, the location and potential displacement of rib fractures of this 3D model. He commented that, "In the future the production of $3 \mathrm{D}$ printed models will have a major role in planning complex chest wall surgery".

$3 \mathrm{D}$ printing is only in its humble beginnings, but this project shows that further research into this topic would be greatly beneficial for this field of medicine. Future research could focus on assessing 3D model printing's efficacy for pre-operative preparation of rib stabilization for surgeons in comparison to the prior modalities of imaging. As previously mentioned the emergence of minimally invasive techniques for surgical rib fixation has shown excellent promise for the invasive treatment of ribs, however no valid imaging technique has been chosen to use with this surgical procedure, therefore investigation into the role of 3D imaging in this field in comparison to the conservative $2 \mathrm{D}$ radiology could be valuable.

\section{Limitations}

This study had a small sample size meaning that the results produced are not as significant as if there was a larger study population. Similarly, this study does not take into account any rib fractures that were missed i.e. those that did have rib fractures but were missed both on chest X-ray and 2D CT. However this would only be a small group and would generally only be those with clinically minor injuries. In assessing the amount of rib fractures recorded on chest X-ray and 2D CT the project relied on the radiologist's report and on the cardiothroacic surgeon's ability in identification for 3D CT. This means there was a difference in reporting between the three modalities which prevented standardisation but this was for reasons as previously stated before. The complications recorded where the acute complications, other than mortality, rather than delayed secondary pathologies.

\section{Conclusion}

This study's aim was to compare the efficacy of $3 \mathrm{D}$ imaging to standard 2D radiology in the identification of rib fractures. It consisted of 22 patients where the appropriate radiology was assessed and compared to each other. It was found that 3D imaging was superior in the detection of rib fractures compared to chest X-rays but was ultimately equal to $2 \mathrm{D} \mathrm{CT}$. It was seen however that $3 \mathrm{D}$ imaging provided a greater depth of detail of the rib fracture compared to $2 \mathrm{D}$ imaging. This projects supports the recommendation of the use of 3D CT in the assessment and pre-surgical preparation for surgical interventions for rib fractures. 3D imaging, either from $3 \mathrm{D}$ CTs to $3 \mathrm{D}$ printing, is an emerging modality that could enhance the visualisation of the complex anatomy of the body.

\section{Appendix}

\section{Appendix 1: 3D Model Printing Information}

The machine used was a Reprap open source 3D printer. The model was a Prusa i3, which costs $£ 140$. The material used was ABS plastic and one reel of the filament cost $£ 7-10$ depending on colour used. The software used was Invesalius to take the CT Dicoms from the PACS and to make the 3D models. Meshlab was used to make the 3D models suitable to print and Matter Control program was utilised to print the models.

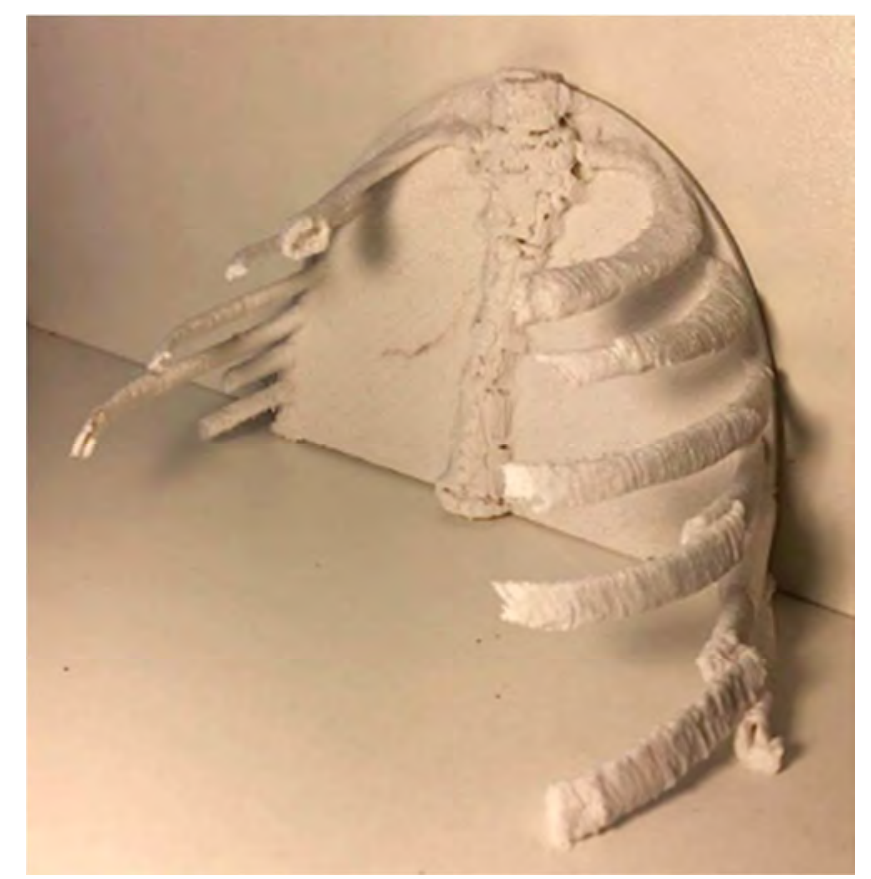

Figure 7. 3D Printed-Model. This is a photograph of the printed model of a patient from the study. A rib fracture can be seen in the $2^{\text {nd }}, 3^{\text {rd }}, 4^{\text {th }}, 5^{\text {th }}$ right ribs.

\section{Appendix 2: Paired T-test}

Paired T-Test and CI: CXR, 2D

Paired T for CXR - 2D 


$\begin{array}{llllllllll} & \mathrm{N} & \text { Mean } & \text { StDev } & \text { SE Mean } & & \text { N } & \text { Mean } & \text { StDev } & \text { SE Mean } \\ \text { CXR } & 22 & 1.8182 .108 & 0.449 & & \text { CXR } & 22 & 1.818 & 2.108 & 0.449 \\ \text { 2D } & 22 & 5.318 & 3.822 & 0.815 & \text { 3D } & 22 & 6.136 & 4.465 & 0.952 \\ \text { Difference } & 22 & -3.500 & 3.569 & 0.761 & \text { Difference } & 22 & -4.32 & 4.71 & 1.01\end{array}$

95\% CI for mean difference: $(-5.082,-1.918)$

$\mathrm{T}$-Test of mean difference $=0(\mathrm{vs} \neq 0): \mathrm{T}$-Value $=-4.60 \mathrm{P}-$ Value $=0.000$

Paired T-Test and CI: 3D, 2D

Paired $T$ for 3D - 2D

95\% CI for mean difference: $(-6.41,-2.23)$

$\mathrm{T}$-Test of mean difference $=0(\mathrm{vs} \neq 0)$ : T-Value $=-4.30 \mathrm{P}-$ Value $=0.000$

\section{Appendix 3: One-way ANOVA}

$\begin{array}{lllll} & \mathrm{N} & \text { Mean } & \text { StDev } & \text { SE Mean } \\ 3 \mathrm{D} & 22 & 6.136 & 4.465 & 0.952 \\ \text { 2D } & 22 & 5.318 & 3.822 & 0.815 \\ \text { Difference } & 22 & 0.818 & 2.702 & 0.576\end{array}$

95\% CI for mean difference: $(-0.380,2.016)$

$\mathrm{T}$-Test of mean difference $=0(\mathrm{vs} \neq 0)$ : $\mathrm{T}$-Value $=1.42 \mathrm{P}$ Value $=0.170$

Paired T-Test and CI: CXR, 3D

Paired $\mathrm{T}$ for CXR - 3D

\section{Method}

Null hypothesis

Alternative hypothesis

Significance level
All means are equal

At least one mean is different $\alpha=0.05$

Equal variances were assumed for the analysis.

Factor Information

$\begin{array}{lll}\text { Factor } & \text { Levels } & \text { Values } \\ \text { Factor } & 3 & \text { CXR, 3D, 2D }\end{array}$

Analysis of Variance

$\begin{array}{llllllll}\text { Source } & \text { DF } & \text { Seq SS } & \text { Contribution } & \text { Adj SS } & \text { Adj MS } & \text { F-Value } & \text { P-Value } \\ \text { Factor } & 2 & 231.5 & 22.04 \% & 231.5 & 115.74 & 8.91 & 0.000 \\ \text { Error } & 63 & 818.6 & 77.96 \% & 818.6 & 12.99 & & \\ \text { Total } & 65 & 1050.1 & 100.00 \% & & & \end{array}$

Model Summary

$\begin{array}{lllll}\text { S } & \text { R-sq } & \text { R-sq (adj) } & \text { PRESS } & \text { R-sq (pred) } \\ 3.60475 & 22.04 \% & 19.57 \% & 898.458 & 14.44 \% \\ \text { Means } & & & & \\ \text { Factor } & \mathrm{N} & \text { Mean } & \text { StDev } & 95 \% \text { CI } \\ \text { CXR } & 22 & 1.818 & 2.108 & (0.282,3.354) \\ \text { 3D } & 22 & 6.136 & 4.465 & (4.601,7.672) \\ \text { 2D } & 22 & 5.318 & 3.822 & (3.782,6.854)\end{array}$

Pooled StDev $=3.60475$

Appendix 4: Crosstabulation for Kappa Statistics for 3D CT Versus Chest X-Ray

Table 1. Number of X-ray Fractures * Number of 3D Fractures Noted Crosstabulation.

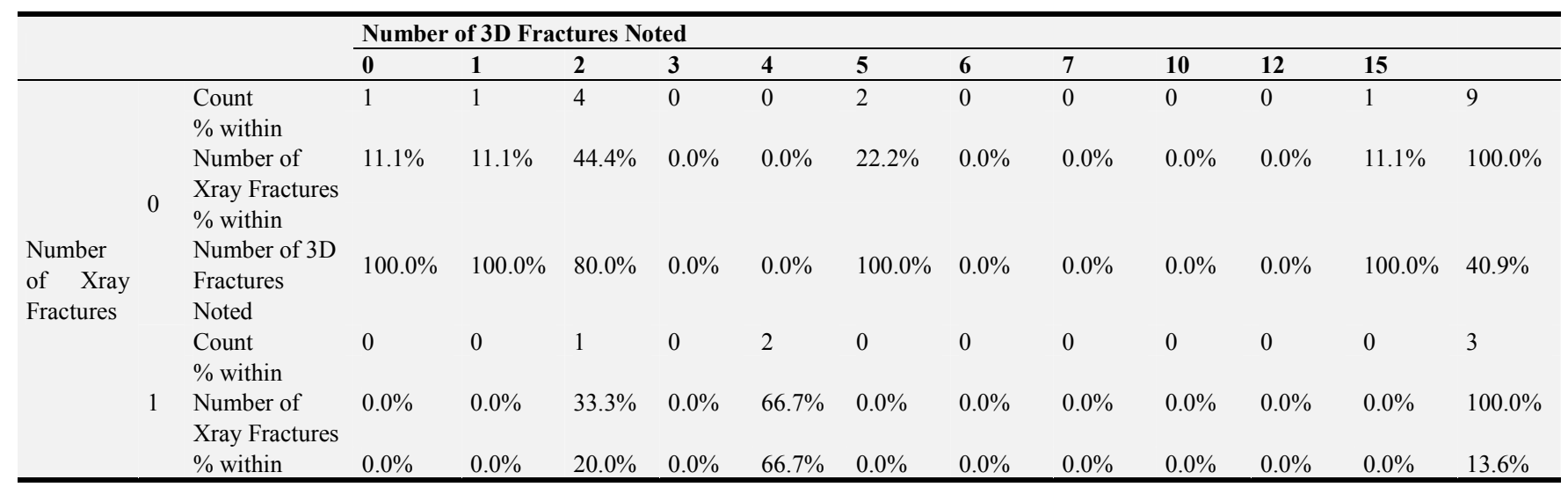




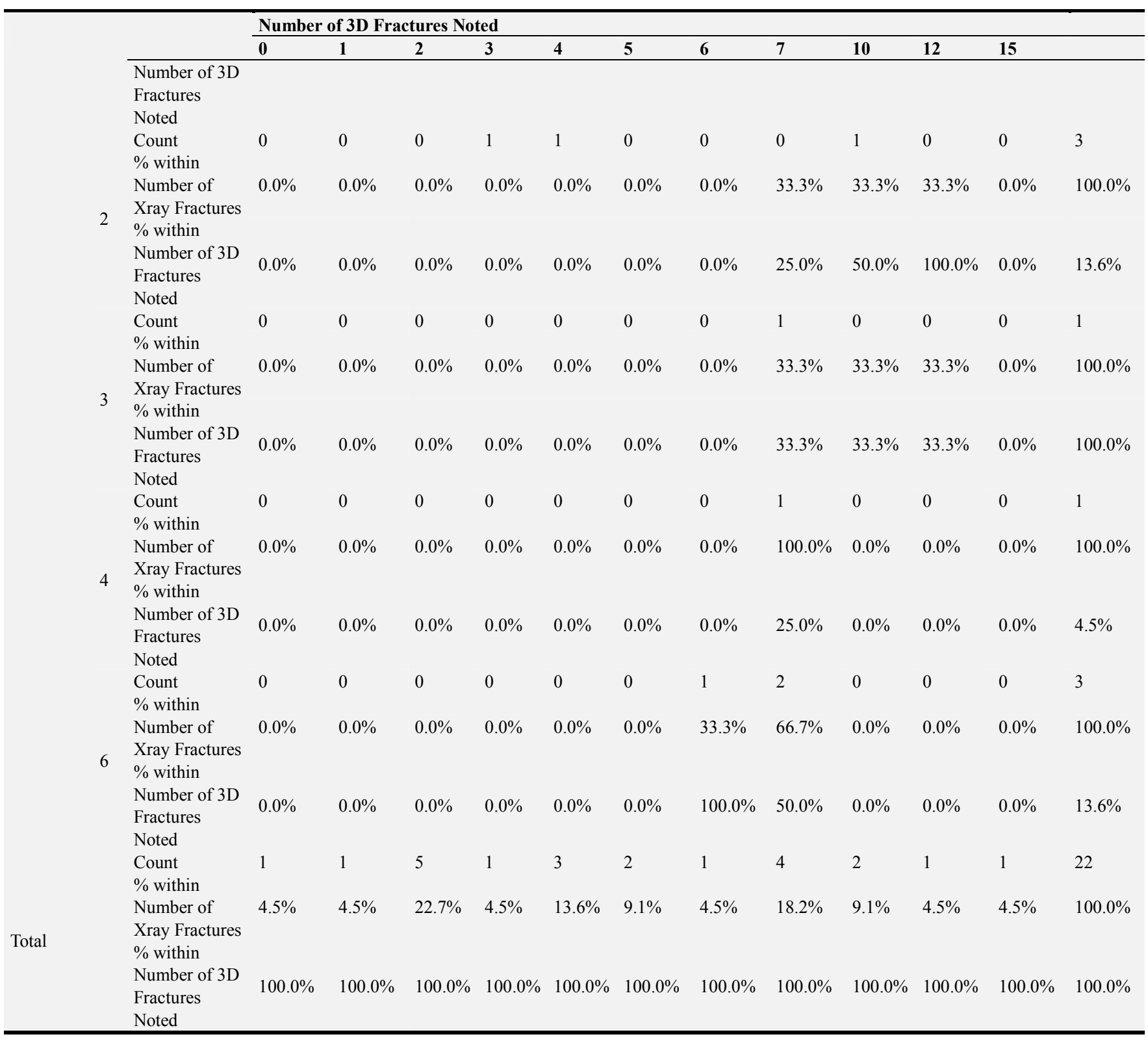

Appendix 5: Crosstabulation for Kappa Statistics for 2D CT Versus $3 D$ CT

Table 2. Number of 2D CT Fractures Noted * Number of 3D Fractures Noted Crosstabulation

\begin{tabular}{|c|c|c|c|c|c|c|c|c|c|c|c|c|c|c|}
\hline & & & \multicolumn{11}{|c|}{ Number of 3D Fractures Noted } & \multirow{2}{*}{ Total } \\
\hline & & & $\mathbf{0}$ & 1 & 2 & 3 & 4 & 5 & 6 & 7 & 10 & 12 & 15 & \\
\hline \multirow{14}{*}{$\begin{array}{l}\text { Number } \\
\text { of } 2 \mathrm{D} \text { CT } \\
\text { Fractures } \\
\text { Noted }\end{array}$} & \multirow{3}{*}{1.00} & Count & 0 & 0 & 1 & 1 & 0 & 0 & 0 & 0 & 0 & 0 & 0 & 2 \\
\hline & & $\%$ within & $0.0 \%$ & $0.0 \%$ & $50.0 \%$ & $50.0 \%$ & $0.0 \%$ & $0.0 \%$ & $0.0 \%$ & $0.0 \%$ & $0.0 \%$ & $0.0 \%$ & $0.0 \%$ & $100.0 \%$ \\
\hline & & $\%$ within & $0.0 \%$ & $0.0 \%$ & $20.0 \%$ & $100.0 \%$ & $0.0 \%$ & $0.0 \%$ & $0.0 \%$ & $0.0 \%$ & $0.0 \%$ & $0.0 \%$ & $0.0 \%$ & $9.1 \%$ \\
\hline & \multirow{3}{*}{2.00} & Count & 0 & 0 & 4 & 0 & 0 & 0 & 0 & 0 & 0 & 0 & 0 & 4 \\
\hline & & $\%$ within & $0.0 \%$ & $0.0 \%$ & $100.0 \%$ & $0.0 \%$ & $0.0 \%$ & $0.0 \%$ & $0.0 \%$ & $0.0 \%$ & $0.0 \%$ & $0.0 \%$ & $0.0 \%$ & $100.0 \%$ \\
\hline & & $\%$ within & $0.0 \%$ & $0.0 \%$ & $80.0 \%$ & $0.0 \%$ & $0.0 \%$ & $0.0 \%$ & $0.0 \%$ & $0.0 \%$ & $0.0 \%$ & $0.0 \%$ & $0.0 \%$ & $18.2 \%$ \\
\hline & \multirow[t]{2}{*}{3.00} & $\%$ within & $100.0 \%$ & $0.0 \%$ & $0.0 \%$ & $0.0 \%$ & $0.0 \%$ & $0.0 \%$ & $0.0 \%$ & $0.0 \%$ & $0.0 \%$ & $0.0 \%$ & $0.0 \%$ & $100.0 \%$ \\
\hline & & $\%$ within & $100.0 \%$ & $0.0 \%$ & $0.0 \%$ & $0.0 \%$ & $0.0 \%$ & $0.0 \%$ & $0.0 \%$ & $0.0 \%$ & $0.0 \%$ & $0.0 \%$ & $0.0 \%$ & $4.5 \%$ \\
\hline & \multirow{3}{*}{4.00} & Count & 0 & 0 & 0 & 0 & 2 & 0 & 0 & 1 & 0 & 0 & 0 & 3 \\
\hline & & $\%$ within & $0.0 \%$ & $0.0 \%$ & $0.0 \%$ & $0.0 \%$ & $66.7 \%$ & $0.0 \%$ & $0.0 \%$ & $33.3 \%$ & $0.0 \%$ & $0.0 \%$ & $0.0 \%$ & $100.0 \%$ \\
\hline & & $\%$ within & $0.0 \%$ & $0.0 \%$ & $0.0 \%$ & $0.0 \%$ & $66.7 \%$ & $0.0 \%$ & $0.0 \%$ & $25.0 \%$ & $0.0 \%$ & $0.0 \%$ & $0.0 \%$ & $13.6 \%$ \\
\hline & \multirow[b]{2}{*}{5.00} & Count & 0 & 0 & 0 & 0 & 1 & 1 & 0 & 1 & 0 & 0 & 0 & 3 \\
\hline & & $\%$ within & $0.0 \%$ & $0.0 \%$ & $0.0 \%$ & $0.0 \%$ & $33.3 \%$ & $33.3 \%$ & $0.0 \%$ & $33.3 \%$ & $0.0 \%$ & $0.0 \%$ & $0.0 \%$ & $100.0 \%$ \\
\hline & 6.00 & Count & 0 & 0 & 0 & 0 & 0 & 0 & 1 & 0 & 0 & 0 & 0 & 1 \\
\hline
\end{tabular}




\begin{tabular}{|c|c|c|c|c|c|c|c|c|c|c|c|c|c|c|}
\hline & & & \multicolumn{11}{|c|}{ Number of 3D Fractures Noted } & \multirow{2}{*}{ Total } \\
\hline & & & 0 & 1 & 2 & 3 & 4 & 5 & 6 & 7 & 10 & 12 & 15 & \\
\hline & & $\%$ within & $0.0 \%$ & $0.0 \%$ & $0.0 \%$ & $0.0 \%$ & $0.0 \%$ & $0.0 \%$ & $100.0 \%$ & $0.0 \%$ & $0.0 \%$ & $0.0 \%$ & $0.0 \%$ & $100.0 \%$ \\
\hline & & $\%$ within & $0.0 \%$ & $0.0 \%$ & $0.0 \%$ & $0.0 \%$ & $0.0 \%$ & $0.0 \%$ & $100.0 \%$ & $0.0 \%$ & $0.0 \%$ & $0.0 \%$ & $0.0 \%$ & $4.5 \%$ \\
\hline & \multirow{4}{*}{7.00} & Count & 0 & 0 & 0 & 0 & 0 & 0 & 0 & 1 & 0 & 0 & 0 & 1 \\
\hline & & $\%$ within & $0.0 \%$ & $0.0 \%$ & $0.0 \%$ & $0.0 \%$ & $0.0 \%$ & $0.0 \%$ & $0.0 \%$ & $100.0 \%$ & $0.0 \%$ & $0.0 \%$ & $0.0 \%$ & $100.0 \%$ \\
\hline & & $\%$ within & $0.0 \%$ & $0.0 \%$ & $0.0 \%$ & $0.0 \%$ & $0.0 \%$ & $0.0 \%$ & $0.0 \%$ & $25.0 \%$ & $0.0 \%$ & $0.0 \%$ & $0.0 \%$ & $4.5 \%$ \\
\hline & & Count & 0 & 1 & 0 & 0 & 0 & 0 & 0 & 1 & 0 & 0 & 0 & 2 \\
\hline & \multirow[t]{3}{*}{8.00} & $\%$ within & $0.0 \%$ & $50.0 \%$ & $0.0 \%$ & $0.0 \%$ & $0.0 \%$ & $0.0 \%$ & $0.0 \%$ & $50.0 \%$ & $0.0 \%$ & $0.0 \%$ & $0.0 \%$ & $100.0 \%$ \\
\hline & & $\%$ within & $0.0 \%$ & $100.0 \%$ & $0.0 \%$ & $0.0 \%$ & $0.0 \%$ & $0.0 \%$ & $0.0 \%$ & $25.0 \%$ & $0.0 \%$ & $0.0 \%$ & $0.0 \%$ & $9.1 \%$ \\
\hline & & Count & 0 & 0 & 0 & 0 & 0 & 0 & 0 & 0 & 1 & 0 & 0 & 1 \\
\hline & \multirow[t]{3}{*}{11.00} & $\%$ within & $0.0 \%$ & $0.0 \%$ & $0.0 \%$ & $0.0 \%$ & $0.0 \%$ & $0.0 \%$ & $0.0 \%$ & $0.0 \%$ & $100.0 \%$ & $0.0 \%$ & $0.0 \%$ & $100.0 \%$ \\
\hline & & $\%$ within & $0.0 \%$ & $0.0 \%$ & $0.0 \%$ & $0.0 \%$ & $0.0 \%$ & $0.0 \%$ & $0.0 \%$ & $0.0 \%$ & $50.0 \%$ & $0.0 \%$ & $0.0 \%$ & $4.5 \%$ \\
\hline & & Count & 0 & 0 & 0 & 0 & 0 & 0 & 0 & 0 & 0 & 1 & 0 & 1 \\
\hline & \multirow[t]{3}{*}{12.00} & $\%$ within & $0.0 \%$ & $0.0 \%$ & $0.0 \%$ & $0.0 \%$ & $0.0 \%$ & $0.0 \%$ & $0.0 \%$ & $0.0 \%$ & $0.0 \%$ & $100.0 \%$ & $0.0 \%$ & $100.0 \%$ \\
\hline & & $\%$ within & $0.0 \%$ & $0.0 \%$ & $0.0 \%$ & $0.0 \%$ & $0.0 \%$ & $0.0 \%$ & $0.0 \%$ & $0.0 \%$ & $0.0 \%$ & $100.0 \%$ & $0.0 \%$ & $4.5 \%$ \\
\hline & & Count & 0 & 0 & 0 & 0 & 0 & 1 & 0 & 0 & 0 & 0 & 0 & 1 \\
\hline & \multirow[t]{3}{*}{13.00} & $\%$ within & $0.0 \%$ & $0.0 \%$ & $0.0 \%$ & $0.0 \%$ & $0.0 \%$ & $100.0 \%$ & $0.0 \%$ & $0.0 \%$ & $0.0 \%$ & $0.0 \%$ & $0.0 \%$ & $100.0 \%$ \\
\hline & & $\%$ within & $0.0 \%$ & $0.0 \%$ & $0.0 \%$ & $0.0 \%$ & $0.0 \%$ & $50.0 \%$ & $0.0 \%$ & $0.0 \%$ & $0.0 \%$ & $0.0 \%$ & $0.0 \%$ & $4.5 \%$ \\
\hline & & Count & 0 & 0 & 0 & 0 & 0 & 0 & 0 & 0 & 1 & 0 & 1 & 2 \\
\hline & \multirow[t]{2}{*}{15.00} & $\%$ within & $0.0 \%$ & $0.0 \%$ & $0.0 \%$ & $0.0 \%$ & $0.0 \%$ & $0.0 \%$ & $0.0 \%$ & $0.0 \%$ & $50.0 \%$ & $0.0 \%$ & $50.0 \%$ & $100.0 \%$ \\
\hline & & $\%$ within & $0.0 \%$ & $0.0 \%$ & $0.0 \%$ & $0.0 \%$ & $0.0 \%$ & $0.0 \%$ & $0.0 \%$ & $0.0 \%$ & $50.0 \%$ & $0.0 \%$ & $100.0 \%$ & $9.1 \%$ \\
\hline & & Count & 1 & 1 & 5 & 1 & 3 & 2 & 1 & 4 & 2 & 1 & 1 & 22 \\
\hline \multirow[t]{2}{*}{ Total } & & $\%$ within & $4.5 \%$ & $4.5 \%$ & $22.7 \%$ & $4.5 \%$ & $13.6 \%$ & $9.1 \%$ & $4.5 \%$ & $18.2 \%$ & $9.1 \%$ & $4.5 \%$ & $4.5 \%$ & $100.0 \%$ \\
\hline & & $\%$ within Number & $100.0 \%$ & $100.0 \%$ & $100.0 \%$ & $100.0 \%$ & $100.0 \%$ & $100.0 \%$ & $100.0 \%$ & $100.0 \%$ & $100.0 \%$ & $100.0 \%$ & $100.0 \%$ & $100.0 \%$ \\
\hline
\end{tabular}

\section{Appendix 6: Kappa Coefficients}

Table 3. 3D CT versus Chest X-ray.

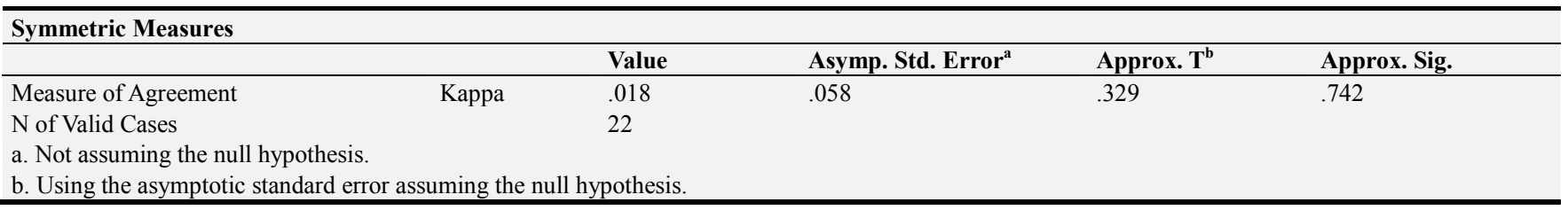

Table 4. 2D CT versus 3D CT- Number of Fractures.

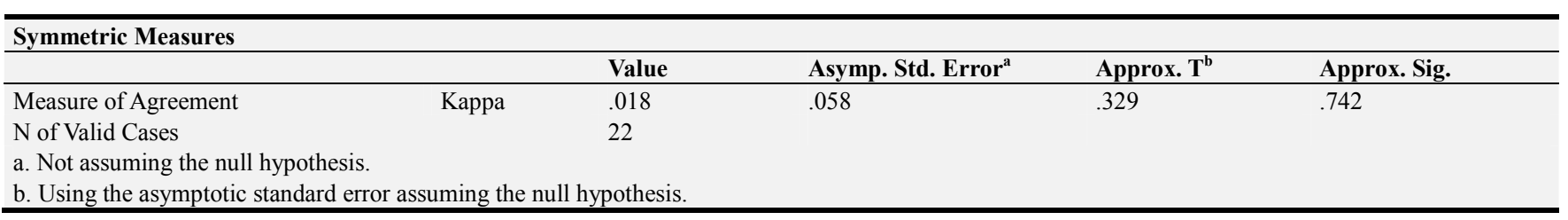

Table 5. $2 D$ CT versus $3 D$ CT-Displacement.

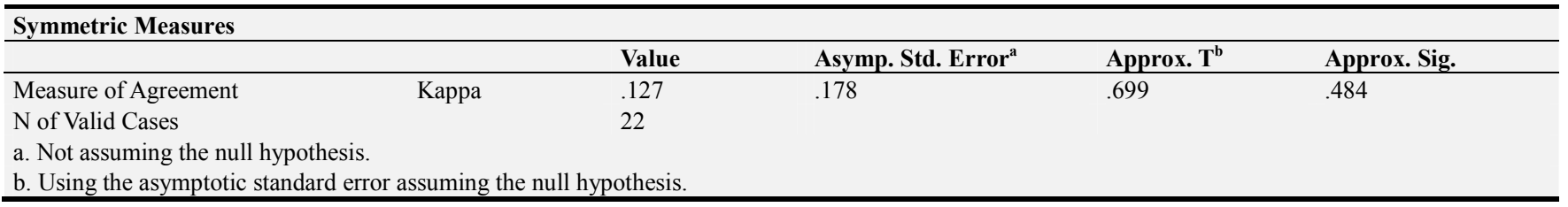

\section{References}

[1] Marasco, S. Lee G., Summerhayes R., Fitzgerald M., Bailey M. (2015). "Quality Of Life After Major Trauma With Multiple Rib Fractures". Injury 46.1: 61-65.

[2] Marasco, S. and Pankaj S.. (2015). "Surgical Rib Fixation Technical Aspects". Injury 46.5, 929-932.

[3] Pulley B., Taylor B., Fowler T., Dominguez N., Trinh T. (2015)."Utility Of Three-Dimensional Computed Tomography
For The Surgical Management Of Rib Fractures". Journal of Trauma and Acute Care Surgery 78.3: 530-534.

[4] Battle, C. and Evans P. (2015). "Predictors Of Mortality In Patients With Flail Chest: A Systematic Review". Emergency Medicine Journal 32.12, 961-965.

[5] May, L, Hillermann C., and Patil S. (2015). "Rib Fracture Management". BJA Education 16.1, 26-32.

[6] Bemelman M., Poeze M., Blokhuis T. J., and Leenen L. (2010). "Historic Overview Of Treatment Techniques For Rib Fractures And Flail Chest". European Journal of Trauma and Emergency Surgery 36.5, 407-415. 
[7] Hoyt, J. (1901). "An Apparatus For Artificial Respiration And For Other Purposes". The Journal of Physiology 27.1-2, 4852 .

[8] Jones T., Richardson E. (1926)."Traction on the sternum in the treatment of multiple fractured ribs." Surg Gynec Obstet. 42: 283 .

[9] Bille, A., Okiror L., Karenovics W., Routledge T. (2012). "Experience With Titanium Devices For Rib Fixation And Coverage Of Chest Wall Defects". Interactive CardioVascular and Thoracic Surgery 15.4, 588-595.

[10] Schuurmans, J., Goslings J., and Schepers T. (2016). "Operative Management Versus Non-Operative Management Of Rib Fractures In Flail Chest Injuries: A Systematic Review". Eur J Trauma Emerg Surg, pp 1-6.

[11] Livingston, D., Shogan B., John P., Lavery R. (2008). "CT Diagnosis Of Rib Fractures And The Prediction Of Acute Respiratory Failure". The Journal of Trauma: Injury, Infection, and Critical Care 64.4, 905-911.

[12] Kaur, J. and Chopra R. (2010). "Three Dimensional CT Reconstruction For The Evaluation And Surgical Planning Of Mid Face Fractures: A 100 Case Study". Journal of Maxillofacial and Oral Surgery 9.4, 323-328.

[13] Esses, S. Berman, P., Bloom, A., Sosna, J. (2011). "Clinical Applications Of Physical 3D Models Derived From MDCT
Data And Created By Rapid Prototyping". American Journal of Roentgenology 196.6, 683-688.

[14] McHugh, M. (2012). "Interrater Reliability: The Kappa Statistic". Biochemia Medica, 276-282.

[15] Fabricant, L., Ham B., Mullins R., Mayberry J., et al. (2013). "Prolonged Pain And Disability Are Common After Rib Fractures". The American Journal of Surgery 205.5, 511-516.

[16] Monkhouse, S. (2007) Clinical Anatomy. 2nd edition, (Edinburgh: Churchill Livingstone Print).

[17] Slobogean, G., MacPherson C., Sun T., Pelletier M., Hameed S. (2013). "Surgical Fixation Vs Nonoperative Management Of Flail Chest: A Meta-Analysis". Journal of the American College of Surgeons 216.2, 302-311.

[18] Pieracci, F. et al. (2016). "A Prospective, Controlled Clinical Evaluation Of Surgical Stabilization Of Severe Rib Fractures". Journal of Trauma and Acute Care Surgery 80.2, 187-194.

[19] Marasco, S., Davies A., Cooper J., Varma D., Bennett V., Nevill R., Lee G., Bailey M., Fitzgerald M. (2013). "Prospective Randomized Controlled Trial Of Operative Rib Fixation In Traumatic Flail Chest". Journal of the American College of Surgeons 216.5, 924-932.

[20] Al Ali A., Griffin M., Butler P. (2015). "Three-Dimensional Printing Surgical Applications", ePlasty Open Access Journal of Plastic Surgery, 15. e37, 352-367. 